\title{
El profesorado y las familias ante el espejo. Demandas en un entorno cambiante y mercantilizado
}

\author{
Teaching staff and families in front of the mirror. \\ Demands in a changing and commodified environment
}

\author{
Joaquín Giró Miranda, Sergio Andrés Cabello'
}

\section{Resumen}

Las políticas de rendición de cuentas son una realidad que ha alcanzado al sistema educativo. Este proceso es fundamental en una sociedad democrática, basada en la participación, la transparencia y la confianza entre los diferentes agentes. Pero también puede dar lugar a su uso como un servicio desde una perspectiva mercantilista considerando la educación como un bien de consumo más. Este artículo cuenta con el objetivo de relacionar la rendición de cuentas, el profesorado y las familias con hijos en educación Primaria y Secundaria. Partiendo de una metodología cualitativa, a través de entrevistas a la comunidad educativa, especialmente madres y padres y profesorado, se ha podido establecer el discurso que se destila desde ambos colectivos en relación a la rendición de cuentas y sus consecuencias. Entre los principales resultados, puede destacarse la existencia de un arco de visiones que van desde la concepción de la rendición de cuentas como una forma de participación, de transparencia y de confianza, hasta otras donde prima más una relación basada en mecanismos de «cuasimercado» y de exigencia de resultados a la institución escolar y sus profesionales.

\section{Palabras clave}

Rendición de cuentas, profesorado, familias, participación, neoliberalismo.

\section{Abstract}

Accountability policies have reached the educational system. This process is essential in a democratic society, based on participation, transparency and upon trust between the different actors. But it also can lead to its use from a mercantilist perspective and result in considering education as another consumer good. This article has the aim to link accountability, teaching staff and families. Parents have the right and the duty to participate in school, as it has increased its functions. Thus, families also receive these results and teachers are concerned about them. This research is based on a qualitative methodology, with interviews to the educational community, specially parents and teachers and then, the discourse of both groups about accountability and its consequences is established. The main results are a range of perspectives varying from the conception of the accountability as a kind of participation, of transparency and trust to others in which a relation based on «quasi - market» mechanisms and demand for results to school and its professionals, prevails.

\section{Key words}

Accountability, teaching staff, families, participation, neoliberalism. 


\section{Introducción}

En el plazo transcurrido entre la aprobación de la Ley Orgánica de Educación (LOE, 2006) y la Ley Orgánica de Mejora de la Calidad de la Educación (LOMCE, 2013), que utiliza el término «gestión basada en los resultados», el sistema educativo español ha desarrollado transformaciones en sus políticas generales que afectan a la rendición de cuentas (accountability) porque se sitúan en un proceso de creciente mercantilización de la educación (Saura, 2015).

Desde hace unas décadas, la accountability, o rendición de cuentas, se ha institucionalizado en todos los ámbitos y niveles de los servicios públicos, incluidos los educativos, primando conceptos como la responsabilidad, la confianza, la eficiencia, la eficacia, la transparencia, etc. (Bovens, 2007; Dutercq, 2016). Y en la educación, la rendición de cuentas tiende a ser sinónimo de nuevas formas de regulación basadas en los resultados (Maroy, 2013).

Los procesos de evaluación en la educación han alcanzado una dimensión pública, es decir, que se encuentran en la agenda pública porque la sociedad valora y juzga, estableciendo presión sobre el sistema educativo, especialmente a través de los informes PISA que miden el rendimiento de los estudiantes en una serie de materias; o a través de los informes TALIS que evalúan docencia y profesorado, ambos de la OCDE. Estos procesos se encuentran más desarrollados en el ámbito anglosajón, especialmente en Estados Unidos, donde es un factor determinante en la elección de escuela por parte de las familias (Fullan et al., 2015).

La rendición de cuentas en la educación tiene sus efectos en la profesión docente porque orientan su práctica hacia dicha evaluación (Barrenechea, 2010; Parcecerisa y Verger, 2016). Aunque los sistemas de evaluación son necesarios, generan numerosas dudas y debates acerca de la metodología, su eficacia y aquellos aspectos que se registran en un entorno tan complejo como el educativo (Dumay et al., 2013). También Bolívar señala que «los actuales formatos de medición de resultados centrados en algunos contenidos o competencias, son insuficientes en la sociedad del conocimientor (2013, p. 80). Por su parte, Landini y Trojan indican respecto al Informe TALIS que «es posible observar el carácter subjetivo de las cuestiones y, a la vez, la imputación de la responsabilidad en el profesorado por los resultados del aprendirajes (2014, p. 325). Éste es un aspecto preocupante, más si cabe cuando aún no se dispone de un estatuto docente que marque las directrices y el desarrollo de la profesión en sus diferentes niveles.

El objetivo de este artículo es analizar el impacto de la rendición de cuentas en España del profesorado ante las familias. Las relaciones entre docentes y familias en la rendición de cuentas se basan en los supuestos de transparencia y confianza. Las familias, por su parte, como agentes de la comunidad educativa, también han transformado su relación con la institución escolar y el profesorado. Además de reclamarles más funciones (Dupriez \& Maroy, 2003; Olmedo, 2008; Tiana, 2008; Bolívar, 2013; Dutercq, 2016; Fernández Enguita, 2016), las familias han ido ganando cada vez más conciencia de su papel, aunque también hay una parte de las mismas que se relaciona con la escuela a través de lógicas de «cuasimercado». Esta situación se inserta dentro de procesos más globales, especialmente de carácter neoliberal, de los cuales la educación no ha sido inmune, aunque inicialmente se había quedado fuera de ella (Dutercq, 2016). En este sentido, la rendición de cuentas puede servir como una argumentación y legitimación de ciertas prácticas neoliberales, además de no olvidarse otros cambios generales que «bowever, universal education (and the accompanying process of burocratization), the development of cultural pluralism, and transformations within the job market have produced a destabilization of schools along with a diversification in the kinds of demands made by clients on the school system.» (Dupriez \& Maroy, 2003, p. 376). 
La insistencia de la LOMCE en «resultados académicos», olvida otros resultados educativos y sociales que después no se miden en las pruebas externas pese a ser una demanda de las familias y del profesorado, como el clima escolar, la integración, el cumplimiento, etc. En definitiva, no se evalúa las competencias y la capacidad de ser un buen ciudadano, sino la eficiencia y la productividad que son exigencias del cuasimercado de la educación.

Cuando hablamos de «cuasimercado» nos referimos a aquellos ensayos del sector público para utilizar los mecanismos de mercado como un modo de mejorar los niveles de eficiencia y productividad de los servicios que presta a la ciudadanía. Estos mercados internos se apoyan en los estímulos de la competencia para mejorar su productividad, haciendo uso de una mejora en la calidad o en la cantidad de los servicios, pero acompañados por una reducción de los costes en la producción de esos servicios, lo que nos introduciría en el concepto de políticas neoliberales de rendición de cuentas frente a las políticas sociales de educación que persiguen principalmente la equidad y la igualdad

Sin embargo, no debe olvidarse que en la rendición de cuentas también se inserta una concepción global de la educación, dentro de una lógica democrática (Dutercq, 2016), donde intervienen todos los integrantes de la comunidad educativa, y donde «la participación de los padres-madres (...) tiene también la perspectiva de lógica correspondencia democrática, por cuanto son los usuarios indirectos de la escuela y además se considera directamente un factor de calidad para el sistema». (Sarramona i López y Rodríguez Neira, 2010, p. 6). Además, como indica Tiana (2008): «la participación democrática en el control del sistema educativo se convierte en una exigencia insoslayable. Ya no es la Administración la única competente para controlar la vida escolar, sino que los ciudadanos y las sociedades deben tomar parte en dicha tarea. A ello se hacia mención al hablar de un control y una gestión participativar (p. 279). Y Silveira insiste en que «los ciudadanos tienen el derecho a participar en los asuntos generales que les conciernen más directamente. Al respecto, los centros escolares fueron pensados como espacios donde se pondría en práctica una democracia más cercana a los ciudadanos». (2016, p. 19).

Aunque se inserta dentro de una lógica democrática, de transparencia y confianza, «el conocimiento del grado de logro de los objetivos de un sistema educativo es una tarea que, en democracia, compete a todos los ciudadanos. Por otra parte, dicho conocimiento es fundamental para hacer el mejor uso de los recursos disponibles y para tomar las decisiones más adecuadas» (Tiana, 2008, p. 280), aunque también tiene un reverso vinculado a la mercantilización de la sociedad (Papadopoulos, 2010). En efecto, los sistemas de evaluación son empleados para institucionalizar una lógica de mercado, distorsionando esa visión democrática y positiva de la accountability: «La responsabilidad se entiende ahora como rendición de cuentas mediante el establecimiento de estándares, provocando -en contrapartida - una (re)centralización del sistema. La creciente presión por los resultados, la libre concurrencia entre las escuelas por conseguir alumnos, con la consiguiente competencia por la clientela, está llevando, en efecto, a que las escuelas se vean obligadas a mejorar de modo continuo» (Bolívar, 2013, p. 66). Y es porque en una lógica de «cuasimercado», se establecen roles diferenciados entre clientes y proveedores de servicios, siguiendo la tendencia neoliberal que se está generalizando en el conjunto de la sociedad y sus instituciones, donde la educación no ha quedado al margen (Olmedo, 2008, Viñao, 2012; Merchán, 2012, Ball, 2014).

\section{Metodología}

Los resultados presentados en este artículo proceden del Proyecto de Investigación "Familias y escuelas. Discursos y prácticas cotidianas sobre la participación en la educación obligatoria" (referencia: EDU2012-32657) de la convocatoria 2012 del Subprograma de Proyectos de Investigación Fundamental no Orientada del Ministerio de Economía y Competitividad. Este proyecto ha analizado las relaciones 
entre familias y escuelas considerando, entre otros aspectos, el discurso de la comunidad educativa acerca de la participación en cuatro Comunidades Autónomas: Cataluña, Aragón, Islas Baleares y La Rioja.

Un equipo interdisciplinar, formado por sociólogos de la educación, pedagogos, trabajadores sociales y psicólogos, llevó a cabo un extenso trabajo de campo cualitativo en treinta y un centros de estas regiones, realizando una etnografía de cada uno de ellos basada fundamentalmente en técnicas como la observación participante y en entrevistas en profundidad con equipos directivos, docentes, madres y padres, personal no docente de los centros y otros informantes clave.

En una primera fase se analizó la documentación de las administraciones educativas y de las federaciones de Asociaciones de Madres y Padres de Alumnos (AMPA); a continuación se realizaron entrevistas mediante un guión semiestructurado a representantes de equipos directivos de centros de educación y de AMPAs. Finalmente, en una segunda fase de carácter etnográfico, se llevó a cabo la observación participante y la realización de entrevistas semiestructuradas, que nos permitieron profundizar en la dinámica de relación existente en lo que tipificamos como centros de éxito; es decir, que tuvieran indicadores de innovación respecto a la participación de las familias, o bien que se significaran por su éxito en esta cuestión. En síntesis, el proyecto abordó la temática de la participación familiar desde una perspectiva básicamente cualitativa.

De este modo, tras el previo análisis de la documentación existente, se llevó a cabo un trabajo empírico durante 2013 con entrevistas en profundidad a cuarenta y seis personas (representantes políticos; de las administraciones autonómicas/locales educativas; del Consejo Escolar autonómico; de federaciones de Asociaciones de Madres y Padres de Alumnos; de sindicatos; de movimientos sociales/educativos y de movimientos de renovación pedagógica), que nos ayudaron a definir el sentido polisémico de la accountability ya que a través de las etnografías surgieron datos relativos a las políticas de rendición de cuentas.

En la fase siguiente, la investigación consistió en una etnografía en treinta y un centros escolares e institutos con una duración mínima de ocho meses en cada uno de ellos (entre el 1 de abril y el 30 de noviembre de 2014; en algún caso ampliado hasta inicios de 2015). Dentro de una muestra de centros seleccionados se contó con las siguientes tipologías: Centros de Educación Infantil y Primaria (CEIPs), Institutos de Educación Secundaria (IES), centros concertados, centros rurales y escuelas unitarias, y centros que experimentan en Comunidades de Aprendizaje. Los criterios de selección de los centros etnografiados estuvieron determinados porque tuvieran indicadores de innovación respecto a la participación de las familias o bien porque se significaran por su éxito en esta temática. Las entrevistas se realizaron a los equipos directivos, el profesorado, y otros profesionales y miembros de juntas directivas de asociaciones de madres y padres.

Para categorizar los datos en el análisis, partimos de la idea de que existen diversas formas de entender la relación entre familias y escuela y distintas dinámicas en los centros escolares (desde aquellas más positivas, que atrapan al menos implicado, a aquellas más negativas, que desaniman al más participativo) y que, aunque son múltiples los factores intervinientes en la creación y consolidación de relaciones positivas, es especialmente relevante la gestión del equipo directivo y su transparencia. Sin negar la importancia de otros factores, observamos que los centros con una gestión más participativa (real, no aparente) y con canales de comunicación entre familia y escuela diversos y transparentes tienen más posibilidades de construir dinámicas de relación positivas. De esta forma hallamos centros con dinámicas positivas de relación que, por el perfil socioeconómico o cultural de las familias (a quienes a menudo se atribuye la 
responsabilidad de que la relación no funcione), podríamos considerar, a priori, que difícilmente las tendrían, y otros que, teniéndolo todo a favor, no las construyen.

\section{El impacto de la rendición de cuentas. Marco Teórico}

El marco teórico en el que nos movemos está estructurado a partir de la participación de las familias en la escuela y su relación con el profesorado, y los procesos de rendición de cuentas en un contexto de cambio social hacia modelos de mercado o «cuasimercado» en la educación.

La participación de las familias en la escuela constituye un fenómeno moderno, garantizado e impulsado desde la legislación educativa, que ha instituido la participación dentro del marco democrático, con la finalidad de que la gestión colectiva de la educación logre el éxito escolar. Además, numerosas investigaciones vienen demostrando la relación positiva entre rendimiento escolar e implicación de las familias (Epstein, 2001; Desforges \& Abouchaar, 2003; Garreta, 2007, 2017). En este proceso democratizador, el papel del profesorado ha resultado fundamental, pues su poder en la gestión de los centros educativos, abriendo o limitando la participación de las familias en la escuela, ha ido en paralelo al cambio de modelos familiares, con su diversa exigencia de resultados y logros escolares.

Por otra parte, el logro y el éxito, la rendición de cuentas y la evaluación, son categorías complejas por la incertidumbre que generan acerca de qué y cómo se valora. De hecho, la accountability se observa como un proceso hacia estándares marcados por quienes diseñan las evaluaciones. En este sentido se asiste a un reduccionismo curricular a través de la creación de estándares, que identifican el éxito escolar con el académico: «Actualmente, en el contexto de una política performativa, las políticas educativas, fuertemente orientadas a incrementar los resultados, tienen como efecto erosionar la implicación de los docentes y debilitar su compromiso». (Bolívar, 2013, p. 66).

También la publicitación de los resultados estandarizados ha llevado a un aumento de la presión sobre los centros educativos y los docentes al establecer rankings dentro de una lógica de «cuasimercado» según las políticas neoliberales de premiar a los mejor situados en dichos rankings (Bolívar, 2013; Dutercq, 2016). De modo que el mercado y la orientación neoliberal en la rendición de cuentas, han determinado todo el proceso, incluyendo la relación con las familias y el conjunto de la comunidad educativa (Kostogriz \& Doecke, 2011).

Algunos cambios observados en la relación entre familias y profesorado devienen de las transformaciones sociales, lo cual se relaciona con ciertos posicionamientos clientelistas, en los que la educación se vincula a un objeto de consumo y donde «se sitúa la relación con la escuela más en términos de exigencia que de confianza y colaboración». (Usategui y Del Valle, 2009, p. 24).

Y en la orientación educativa actual, cada vez más vinculada al mercado, predomina la importancia de los resultados académicos donde se habla de la calidad y la satisfacción de los clientes (Bolívar, 2013). Esto trae consigo el que si bien los mecanismos de rendición de cuentas «tendent surtout réduire l'incertitude et l'opacité des clients/mangers, en vue de favoriser la bonne marche du marché ou de l'organisation productive scolaire, mais au risque d'une perte d'autonomie professionelle des enseignants, et sans pour autant que l'Ecole ne redevienne une institution politique digne de confiancers. (Maroy, 2013, p. 299).

En consecuencia, la política neoliberal (políticas basadas en la búsqueda de beneficios económicos) triunfa sobre la perspectiva igualitarista (políticas basadas en la equidad y la igualdad de oportunidad con independencia de los condicionantes socioeconómicos), al imponer mecanismos de control que permiten la comparación entre centros, discriminándolos. Otro aspecto determinante en ciertos usos de carácter 
neoliberal de la rendición de cuentas es el relativo a la elección de centro. El establecimiento de rankings y la obsesión por los resultados dan lugar a una lógica competitiva que «en este sistema de 'cuasi-mercado' (que se diferencia del puro mercado económico porque no implica un intercambio monetario entre cliente y proveedor), los centros se transforman en autónomos y son inducidos a 'ganar' sus clientes a través de sus iniciativas, su trabajo, su equipo, la calidad de su trabajo y de su oferta educativa». (Maroy, 2008, p. 2).

Olmedo va incluso más a allá e insiste en que «si el centro educativo es considerado como un 'productor' de servicios y las familias como 'consumidoras' de los mismos, definidos ambos de esta forma según el nuevo discurso neoliberal, el ideal de participación y control por parte de las familias sobre el proceso de producción, el proceso educativo, en definitiva, no es otro que la elección del 'proveedor' más acorde con los intereses familiares》. (2008, p. 20). Sin embargo, este proceso no garantizaría la eficacia del sistema educativo, y sí aumentaría la segregación escolar (Betenner et al., 2005; Kostogriz \& Doecke, 2011; Labaree, 2013). Además, mediante esa orientación neoliberal se estaría produciendo una transferencia de la responsabilidad del proceso educativo, exclusivamente a las escuelas y a los docentes, quedando las familias como meros espectadores (Kostogriz \& Doecke, 2011). Quizás, en origen, el profesorado ha vinculado más el sentido de calidad con la prestación de un servicio, acercando las instituciones educativas al mercado de bienes y servicios (Kostogriz \& Doecke, 2011; Bolívar, 2013), mientras las familias se han acomodado a su nueva situación de consumidores de servicios.

Sin embargo, los sistemas de evaluación son entendidos como necesarios para incorporar mecanismos de mejora desde los que conseguir los objetivos programados (Anghel et al., 2013, p. 2) y, por tanto, son aceptados y adoptados por el sistema educativo, cada vez más en concurrencia con los sistemas educativos de otros países. No obstante esta premisa, la accountability también tiene consecuencias negativas sobre los docentes, dentro de una redefinición más amplia y general de sus funciones (Whitty, 2006; DarlingHammond et al., 2014; Day \& Gu, 2012), tanto en su conceptualización profesional como en el aumento del stress, el burnout, etc. (Berryhill et al., 2009), además de afectar a las relaciones con la comunidad, que se ven transformadas por esa rendición de cuentas (Kostogriz \& Doecke, 2011). En este sentido, «estas políticas tienden a otorgar mayores cotas de poder a la administración, los directores o las familias, a la vez que socavan la capacidad de decisión del profesorado. Cuando los docentes perciben que las politicas les desempoderan o restringen su autonomía profesional (por ejemplo, sobre decisiones en el ámbito curricular), éstos generalmente adoptan estrategias de resistencia con la que hacer frente a la aplicación de las reformas». (Parcerisa y Verger, 2016, p. 34).

En consecuencia, nos encontramos ante un conflicto de rol del profesorado mediante un proceso performativo que orienta parte de su labor docente hacia los resultados demandados por las pruebas estandarizadas: «These representations associated with results-based regulation revolve around the twofold idea that, on the one hand, teachers are responsible for the results of their students and, on the other hand, the results of the external evaluation had to serve as an information base to modify their teaching practices.» (Dumay et al., 2013: 107). Y aunque se producen tensiones y resistencias entre los docentes, e incluso disonancias en la aplicación de estas políticas (Hall \& McGinity, 2015; Dutercq, 2016), las presiones a las que se somete al profesorado aumentan, implicando más responsabilidades y exigencias (Usategui y Del Valle, 2009; Parcerisa y Verger, 2016), y es porque no tiene ese margen de confianza de que disponía en épocas anteriores, cuando su figura, su autoridad, no se ponía en entredicho.

\section{Profesorado y familias ante la rendición de cuentas}

En el análisis del discurso del profesorado, si bien las funciones y responsabilidades en el seno de la comunidad educativa están diferenciadas, en razón de la peculiar aportación que realizan al proyecto común tanto 
la entidad titular, como el profesorado, las familias, el alumnado, el personal de la administración y otros agentes colaboradores, lo cierto es que todos participan de la idea genérica de que los centros son empresas donde cada persona ocupa una posición diferenciada y a la que se le adjudican determinadas funciones. Por ejemplo, en el caso de las familias, se quejan de que las entidades educativas no atienden sus ruegos y demandas como partícipes a título de clientes de las mismas:

\section{"Yo aquí lo que veo es que la voz. del padre no se tiene mucho en cuenta. Si tenemos en cuenta cómo está formado, al final es la empresa, el centro, el que al final va a dirigir e ir hacia donde él quiere el Consejo Escolar. Te escuchan, les conviene o no les conviene, pero al final las decisiones están toma- das». (CIPCALR14B)}

Esa afirmación de que en última instancia las decisiones están tomadas por el centro educativo, denota la inexistencia de comunidad donde, a pesar de que formalmente aparecen los instrumentos democráticos para que las decisiones se tomen en régimen de igualdad, aparece en el vocabulario la figura de la empresa y el cliente. La primera toma de decisiones, después de escuchar al cliente, no necesariamente satisface a todos los consumidores, quizás a los más interesados en participar en la gestión de la empresa, los más profesionales (Betebenner et al., 2005). Y es que unos y otros se imaginan como profesionales, de modo que reclaman una relación entre iguales, no subordinada, tal y como se ofrece desde la perspectiva de una relación entre empresa y cliente.

Por otra parte, las familias cuentan con una visión del papel y función que deberían desempeñar el profesorado, en no pocas ocasiones contrapuesto con el manifestado por el profesorado en relación a la rendición de cuentas y los procesos de participación, y con un perfil cada vez más mercantilizado. En este sentido, el ciudadano, antes que ciudadano es consumidor, y la relación es absolutamente clientelar, por lo que una parte significativa de las familias se relacionan con la escuela como consumidores, con las consecuencias que supone para la profesión docente, que se convierte en un mero servicio burocrático cuyo cometido se orienta hacia la satisfacción de las demandas del cliente. Muchas familias ven la educación como un valor de consumo, en parte por el modo de presentarse los propios centros como prestadores de servicios:

"Y quizá nosotros seamos culpables también. Cuando en el colegio se implanta pues hace unos ocho
años el sistema de gestión de calidad en base a una norma ISO, en muchos aspectos, el alumno y las
familias se convierten en un cliente, me da igual que en la escuela pública que privada. Si que es nues-
tro cliente, y le tenemos que dar el mejor servicio, pero en el caso de cómo tenemos que responder a la
demanda de las familias, o en este caso las familias tienen que responder a las demandas que el colegio
bace, que siempre pedimos que las familias vayan de la mano de nosotros, que, en la página web del
colegio, en el apartado Personas es lo que pedimos. Si no vamos de la mano, no vamos a conectar».
(CIPCPS1LR14A)

Y desde el momento en que se afirma esta idea de centro educativo versus empresa, las familias se convierten en clientes que exigen productos determinados (éxito académico), a la vez que demandan cambios en las funciones del profesorado empleado (éxito educativo, padres en la sombra). Es decir, las familias consumidoras de educación como un servicio más de mercado, ya no solo reclaman del profesorado el éxito académico sino que también dan por supuesto que el profesorado debe realizar las funciones de guarda y custodia de sus hijos, así como de preparación para la vida. Es lo que se entiende por ejercer de padres en la sombra.

«Hay muchas veces que hay una relación cliente-empresa, y muchas veces se carga de responsabilidad a los centros de muchas cosas, y muchas cosas, funciones, que los propios padres, en este caso clientes, creen 
que debería tener el colegio, se quejan por cosas por cómo ellos quieren que sea, no vas a una empresa a decir cómo se hagan las cosas. En los colegios pasa a veces esto, que es una cosa extraña, que exigen que se hagan las cosas como ellos quieran no como el centro...». (CIPCO2LR14A)

Efectivamente, tras la exigencia de buenos resultados por parte de las familias-clientes se esconde una pérdida de confianza en la institución escolar y en la profesionalidad del profesorado sobre quienes recaen todas las responsabilidades. De esta manera se produce un doble proceso de desprofesionalización al perder el sentido vocacional, y proletarización al precarizarse la situación laboral.

Cuando los padres se muestran solidarios con el centro, es para trasladar sus conocimientos y experiencias que como profesionales han atesorado, y que el centro bien puede aprovechar. Entre los más comprometidos, existe una conciencia solidaria que les hace aborrecer cualquier límite hacia una actuación que no incorpore el sentido de comunidad; de ahí que nadie tenga claro cuáles son sus funciones, o hasta dónde puede llegar desde su posición en la estructura del centro. Un estereotipo colgado del funcionariado docente es que éste no se encuentra comprometido con la comunidad educativa, y que tan solo se comportan de acuerdo al mandato por el que han sido contratados. Sin embargo, la tónica del discurso docente es que las familias han dejado de lado sus funciones de padres y madres y han delegado en docentes, funcionarios o no, sus responsabilidades para con la educación integral de sus hijos (Giró y Andrés, 2016; Andrés y Giró, 2016b; Giró y Andrés, 2017).

Igualmente, la profesionalidad viene investida de determinadas funciones que limitan la participación de cada uno de los actores o agentes educativos, al punto que si esto no sucede es porque falta o falla la coordinación, función que correspondería, aunque no se cite, a los equipos directivos o directores de centro.

En una virtual estructura piramidal, el profesorado se encontraría al mismo nivel que las familias, y de ahí que se exija una diferenciación en la participación comunitaria, de acuerdo a las funciones que se piensan inherentes al profesional de la educación. Porque si no hay diferenciación en las funciones, y si no se ponen límites a la participación de las familias, el profesorado deja de ser considerado como profesional, o deja de considerarse a sí mismo como profesional de la educación. En definitiva, se reclama un margen de confianza de las familias para que puedan desarrollar aquellas funciones que consideran como profesionales (Llevot y Bernard, 2015). Margen de confianza que equivale a reclamar una autoridad en sus decisiones, que las familias, según su grado de implicación en la tarea educativa, ponen en cuestión desautorizando en ocasiones el modo de actuar del docente.

"Los padres tendrían que darnos margen de confianza, como profesionales, tanto para dar premios como para castigar, castigo me explico, 'oye, tu bijo se ha quedado dos dias sin recreo por esto', 'de acuerdo, me parece perfecto' y no 'ah, y por qué le has dejado, es que no te ha hecho tal, es que le expliqué ayer cómo habia que hacerlo'. Vamos a ver, no tratemos de infravalorar mi tarea, de infravalorar lo que be hecho». (CPUDLR14AI)

Sin embargo, esta situación cambia según el nivel educativo del que estemos tratando, pues si bien en Infantil y Primaria madres y padres se encuentran totalmente volcados en la vida de los centros, cuando sus hijos alcanzan el nivel de Secundaria parecen hacer dejación de sus funciones como educadores, concurriendo en los docentes de este nivel otro tipo de reclamaciones. Así pues, el nivel educativo se ha mostrado como una frontera en los discursos del profesorado, admitiendo a un lado y a otro de esa frontera simbólica, un discurso y unas prácticas radicalmente distintas, tanto de familias como del profesorado, si bien 
encontramos de forma minoritaria a quienes sostienen que la verdadera frontera en la participación se encuentra en el grado de profesionalidad.

En general, los equipos directivos entienden que, en medio de tanta diversidad y heterogeneidad en la composición de las familias, lo que sobresale en la relación con las mismas es la calidad de los encuentros, bien sean estos individuales o colectivos. Incluso uno de los lemas que se han impuesto en los últimos años, sobre todo formando parte de los movimientos sociales en favor de la escuela pública, ha sido hablar de calidad, de una «educación de calidad».

Cuando al profesorado se le pregunta por resultados de su actividad en el seno de la comunidad educativa, siempre entiende que se habla de resultados académicos y, en este sentido, manifiesta que éstos mejoran cuando la participación de las familias se sostiene con una cierta asiduidad y consistencia. Con la participación no solo se consigue uno de los objetivos de la escuela, como es la educación del alumnado y el éxito escolar del alumnado, sino que también se consigue dejar de hablar de familias y profesorado como si de dos bandos enfrentados se trataran, para observarlos como una comunidad educativa en la que todos se sienten integrados y partícipes.

Así pues, la implicación de las familias logra que los resultados sean mejores, y en esto también suele influir el nivel sociocultural y/o económico de los padres. El profesorado señala que hay diferentes perfiles de padres/madres, y que algunos son muy exigentes, que piden mucho en cuestión de resultados académicos. Además, según su parecer, las familias se preocupan solo de los resultados académicos en la confianza de prevenir el fracaso escolar.

Y si de modo generalizado se entiende que una dinámica participativa de las familias produce necesariamente un efecto beneficioso en el éxito educativo y no solo académico del alumno, también una parte del profesorado señala que la implicación de las familias no produce necesariamente unos resultados académicos mejores, y que no existe una correspondencia directa, sobre todo, cuando para muchas familias la asistencia o la relación con la escuela no se observa como una actividad prioritaria si los resultados académicos de sus hijos son favorables. Por contra, donde denotan mayor exigencia de las familias, es precisamente entre aquéllas cuyos hijos obtienen peores resultados. Es como si necesitaran una explicación causal que no radicara en el comportamiento estudiante de sus hijos, pasando la responsabilidad de los resultados al profesorado.

Un trabajo profesional, permite la complicidad de las familias, la cuales observan que los resultados mejoran conforme se implican, cerrando un círculo virtuoso (Bolívar, 2006), pese a que algunos docentes muestran sus dudas acerca de la bondad de la participación de las familias en la obtención de mejores resultados académicos, quizás temerosos de ser blanco de las críticas que las familias trasladan al profesorado, cuando los resultados académicos no son los esperados. En realidad, lo que evidencian es que trasladan la responsabilidad de los malos resultados a las familias, las cuales deberían conocer no solo los objetivos generales del centro, sino estar ahí; es decir, apoyar al docente y no culpabilizarlo. En otros casos, la responsabilidad de los buenos o malos resultados académicos queda salvaguardada, siempre cuando se mantiene una línea de información y comunicación con las familias, de modo que son ellas quienes deciden la resolución a tomar respecto a los resultados académicos de sus hijos.

Sin embargo, en la relación familias y escuelas siempre aparece el discurso de los límites a la participación de las familias, y éstos se establecen básicamente en el control pedagógico, monopolio de los docentes y ajeno al interés de las familias. El problema surge cuando el profesorado se encuentra con aquellas familias 
que, con igual o superior preparación pueden cuestionar su relación pedagógica. En este sentido, las nuevas tecnologías empleadas en la comunicación de familias y escuelas han servido para apaciguar las divergencias en materia de control de los alumnos, y de las medidas a tomar en consonancia a sus actividades. Así, casi todos los centros escolares se han provisto de plataformas digitales a través de las cuales los alumnos son controlados (faltas de asistencia, notas y calificaciones, etc.), y las familias son notificadas sin necesidad de desplazamientos, e indirectamente sin necesidad de encuentros, si bien las tutorías de carácter individual se mantienen, eso sí, a través de citas programadas a través de los mismos canales.

Plataformas que se establecen para control del alumnado, pero que también son un instrumento de control político de los centros por parte de las administraciones educativas, toda vez que las plataformas indican la oferta de materias optativas, la ratio de alumnos por asignatura, etc., de modo que dichas plataformas se han convertido en una especie de Gran Hermano en una sociedad hiperconectada.

De esta forma, y en parte gracias a la llegada de estas nuevas herramientas, el profesorado y los equipos directivos sostienen que con las familias se puede coadyuvar, tender puentes, solicitar su participación y fomentar que vean el centro como su casa, a pesar de que en esa relación de familias-escuelas, todos los centros imponen límites o la supresión de los mismos, y el tratamiento en términos de igualdad o de dominación. El profesorado no se decanta por ninguna opción o más bien es más proclive a la imposición de límites, aunque considera que se debe avanzar en el tratamiento entre iguales.

«Anteriormente, trabajabas y la familia confiaba en tu trabajo e incluso te lo valoraba, en ese sentido. Ahora... se ba convertido un poco en la exigencia, a veces quieran que seas su sustituto, en ese sentido, pero un sustituto de acuerdo con las ideas que yo pueda tener...no es el concepto que teníamos de la Educación de antes». (CIPCPP3LR14A)

Pero, en consonancia con los tiempos actuales, las familias se han vuelto más individualistas, atendiendo únicamente a los problemas específicos de sus hijos/as, sin contextualizarlos en el centro y en colaboración con otras familias. Existe un importante individualismo y egoísmo en la sociedad, que se trasluce en la participación de las familias en la escuela, muy individualizada, que se preocupa tan solo de los resultados académicos del hijo/a, sin dar el paso de integrarse a través de la participación colectiva, que la ven más como un «marrón».

"Queremos que las familias, a modo individualizado y puntualmente, sepan lo que les pasa a sus bijos en el centro, en cuanto a faltas, asistencia o no asistencia, resultados académicos, y que de alguna manera les preocupen las cosas que creemos deben preocuparles». (IPUEDLR14AII)

Y dentro de la exigencia de resultados, cuenta con una especial relevancia el peso de las notas, reforzando esa participación individualizada y centrada en el aspecto académico de los hijos, siendo la tutoría individual la vía de participación más importante. La tutoría para parte de las familias significaría más una preocupación individual por la marcha de sus hijos y un medio de control de su vida escolar. En definitiva, los resultados académicos que se vincularían con conceptos como la «calidad» o la «satisfacción del cliente» (Bolívar, 2013), los cuales provienen del mundo económico, mientras que en la educación entran en juego otros como la equidad, la cohesión social, la libertad, etc., (Sarramona i López y Rodríguez Neira, 2010).

Si los docentes suspenden mucho encontramos otra de las razones por las que las relaciones con las familias son poco colaborativas. Unos y otros se juzgan culpables de una participación negativa. Los pa- 
dres, en vez de pensar que el profesor está de su parte, están a la defensiva, y en vez de ir al centro para hablar y colaborar, se muestran menos participativos.

\section{"Yo siempre les digo a los padres que, cuando los padres están a favor del profesor, el niño sube como la espuma, en comportamiento, en rendimiento, en todo; pero cuando los padres están reticentes o "pues tu profesor no sé qué o no sé cuántos", el niño aprovech a la ventaja y se rebela». (CPUPP3LR14BIII)}

También es cierto que hay un sector del profesorado que no admite las críticas que puedan llegar desde las familias, que temen su sanción, y cuando se ven obligados a mantener comunicación o colaboración con las familias (por ejemplo, en las tutorías) lo hacen desde su criterio, su orden, su norma; es decir, desde una posición de dominio. Aquí es donde se encuentra el talón de la participación, en la coordinación y en la colaboración en términos de igualdad, pero respetando la diversidad.

Hoy día las familias están informadas y quieren opinar. Van perdiendo el miedo a participar en los centros y a colaborar; sin embargo, es una colaboración con fecha de caducidad (a la finalización de los estudios obligatorios), por lo que el compromiso de las familias a largo plazo es más complicado y, si colaboran, siempre es sobre acciones a corto plazo.

\section{Conclusiones}

Los procesos de rendición de cuentas (accountability) son una realidad institucionalizada y reconocida en todos los ámbitos de los servicios públicos, y la educación no es una excepción. Se inserta dentro de una lógica democrática, de transparencia y confianza y, a su vez, se vincula a los procesos de mercantilización de la vida social. En esta doble característica se instala la relación entre la rendición de cuentas, la participación de las familias en la escuela y su impacto en el profesorado. Desde ese modelo democrático en el que la accountability sería necesaria e imprescindible, hasta el modelo donde se desarrollan los procesos de «cuasimercado», entendiendo las familias la educación como un bien de consumo más. Llegados a este extremo, la rendición de cuentas desempeñaría una función legitimadora de esos nuevos modos de organización y control, con consecuencias sobre el profesorado y el concepto de profesión, que una vez desvalorizada y burocratizada se inclina hacia la semiprofesión. Incluso, la propia participación y la visión del profesorado sobre esta implicación de madres y padres estarían orientadas hacia esa rendición de cuentas hacia las familias, como un elemento más de la burocratización de la profesión docente (institucionalización formal y rígida de la participación), que alcanzaría nuevas formas de control con las Tecnologías de la Información y la Comunicación (Giró y Andrés, 2017).

La implementación de lógicas de mercado en la educación y su relación con las familias afecta directamente a conceptos como el compromiso del profesorado y su relación con el conjunto de la comunidad educativa donde se inserta. Esa orientación hacia los resultados claramente definiría cómo interactúan los docentes con madres y padres, que en función de su visión sobre la educación (democrática vs. mercantilista) actuarían como ciudadanos o como clientes. De hecho, hay que destacar que en la investigación desarrollada, equipos directivos y docentes hacían especial hincapié en conceptos como la transparencia, la confianza, la comunicación, la disponibilidad y la bidireccionalidad en su relación con las familias.

Por su parte, el profesorado ha transformado su visión de la profesión, y ese proceso se observa claramente en esa relación de las familias y el feedback que reciben. Primero, porque la rendición de cuentas es identificada de forma única con los resultados académicos, que son los que importan a no pocas familias, especialmente a medida que los estudiantes avanzan en la Educación y que alcanza su punto máximo en 
Secundaria (Giró, et al., 2014). En segundo lugar, porque, al demandar más funciones a la escuela y los profesores, las familias también van a reclamar más resultados, produciéndose un «desborde» de los docentes, superados por la situación que pueden orientar su acción hacia lo que se les reclama. Y, en tercer lugar, dentro la heterogeneidad de situaciones y de familias, así como de docentes, también habrá grupos de madres y padres que por su nivel socioeconómico, identificación cultural con la escuela, o motivaciones, tendrán más capacidad para leer y aprovechar esos resultados de la rendición de cuentas (Betebenner et al., 2005).

Las tendencias de mercantilización de la educación son un hecho en el mundo, y España no es una excepción. El paso de la LOE (2006) a la LOMCE (2013) se inserta dentro de esa lógica, incluso la primera ya contaba con elementos de «cuasimercado» con alusiones directas a conceptos como la calidad (Andrés y Giró, 2016a). La LOMCE va mucho más allá con una concepción neoliberal de la educación que contó con una fuerte contestación social que, aunque no pudo evitar su aprobación, minimizó alguno de sus contenidos como el establecimiento de rankings entre los centros, datos que en España no son públicos (Giró y Andrés, 2014). Pero, la LOMCE también es una ley performativa en ese proceso de mercantilización que ha profundizado en la estandarización de los resultados y en la burocratización de la profesión docente. Con respecto a las familias, a la par que el Consejo Escolar se transforma en un mero órgano consultivo, incide en esa lógica de mercado con un discurso neoliberal (Saura, 2015). Muchas familias, unas por convencimiento y posibilidad, otras por necesidad y las más arrastradas por la corriente, se verán subsumidas en ese proceso.

Finalmente, cuando hablamos de comunidad educativa lo hacemos como un concepto global, donde están presentes todos los actores implicados. Sin embargo, cuando se le piden resultados al sistema educativo, el foco queda fijado en la escuela y los docentes, como si estos fuesen independientes de su entorno, de las familias, de las administraciones, etc. Si comunidad educativa y educación somos todos, la responsabilidad debería ser compartida, pero las corrientes mercantilizadoras, al transformar la educación en un producto, han puesto el foco en el «proveedor de servicios», como se observa en las reacciones que suscitan la publicación de los resultados del Informe PISA o TALIS. La concepción democrática de la educación, y el papel que en la misma deben desempeñar todos los actores, se ve sujeta a una gran cantidad de presiones y tensiones, siendo los primeros perjudicados los estudiantes, y por extensión, los docentes.

\section{Referencias bibliográficas}

Andrés, Sergio y Giró, Joaquín (2016a): "La participación de las familias en la escuela: una cuestión compleja”. Revista de Evaluación de Programas y Políticas Públicas, 7, 28-47. doi: 10.5944/reppp.7.2016.16302

Andrés, Sergio y Giró, Joaquín (2016b): "El papel y la representación del profesorado en la participación de las familias en la escuela". Revista Electrónica Interuniversitaria de Función del Profesorado, 19 (1), 17-29. doi: 10.6018/reifop.19.1.245461

Anghel, Brindusa; Cabrales, Antonio; Sainz, Jorge y Sanz, Ismael (2013). Publicación de los resultados de las pruebas estandarizadas externas: ¿Tiene ello un efecto sobre los resultados escolares? Madrid: Fundación de Estudios de Economía Aplicada (FEDEA), http://documentos.fedea.net/pubs/dt/2013/dt-2013-07.pdf

Ball, Stephen J. (2014): “Globalización, mercantilización y privatización: tendencias internacionales en Educación y Política Educativa”. Archivos Analíticos de Políticas Educativas, 22 (41). doi: 10.14507/epaa. v22n41.2014 
Barrenechea, Ignacio (2010): "Evaluaciones Estandarizadas: Seis Reflexiones Críticas". Archivos Analíticos de Políticas Educativas, 18 (8), 1-27. http://epaa.asu.edu/ojs/article/view/751

Berryhill, Joseph; Linney, Jean Ann \& Fromewick, Jill (2009): "The Effects of Education Accountability on Teachers: Are Policies Too-Stress Provoking for their Own Good". International Journal of Education Policiy \& Leadership, 4(5). http://journals.sfu.ca/ijepl/index.php/ijepl/article/view/99/68

Betebenner, Damian W., Howe, Kenneth R. \& Foster, Samara S. (2005): "On School Choice and TestBased Accountability”. Education Policy Analysis Archives, 13 (41). doi: 10.14507/epaa.v13n41.2005

Bolívar, Antonio (2013): "La lógica del compromiso del profesorado y la responsabilidad de la escuela. Una nueva mirada”. REICE. Revista Iberoamericana sobre Calidad, Eficacia y Cambio en Educación, 11 (2), 60-86. http://www.redalyc.org/pdf/551/55127024003.pdf

Bolívar, Antonio (2006): “Familias y escuelas: dos mundos llamados a trabajar en común”. Revista de Educación, 339, 97-118.

Bovens, Mark (2007): “Analysing and Assessing Accountability: A Conceptual Framework". European Law Journal, 13 (4), 447-468. doi: 10.1111/j.1468-0386.2007.00378.x

Darling-Hammond, Linda; Wilhoit, Gene \& Pittenger, Linda (2014): “Accountability for College and Career Readiness: Developing a New Paradigm". Education policy analysis archives, 22 (86). doi: 10.14507/ epaa.v22n86.2014

Day, Christopher y Gu, Qing (2012). Profesores: vidas nuevas, verdades antiguas. Una influencia decisiva en la vida de los alumnos. Madrid: Narcea.

Desforges, Charles y Abouchaar, Alberto (2003): “The impact of parental involvement, parental support and family education on pupil achievement and adjustment: a literature review". DfES Research Report, 433. http:/ /www.creativitycultureeducation.org/the-impact-of-parental-involvement-parentalsupport-and-family-education-on-pupil-achievement-and-adjustment-a-literature-review

Dumay, Xavier, Cattonar, Branka, Maroy, Christian \& Mangez, Catherine (2013): "The Local Institutionalization of Accountability in Education: Network and Bureaucratic Modes of Implementation". RISE. International Journal of Sociology of Education, 2(2), 99-141. doi: 10.4471/rise.2013.26

Dupriez, Vincent \& Maroy, Christian (2003): "Regulation in school systems: A theoretical analysis of the structural framework of the school system in French-speaking Belgium”. Journal of Education Policy, 18 (4), 375-392.

Dutercq, Yves (2016): “Accountability, una caja de herramientas, no una política: El caso del sistema educativo francés". Profesorado. Revista de currículum y formación del profesorado, 20 (3), 145-169. http://www. redalyc.org/pdf/567/56749100006.pdf

Epstein, Joyce (2001). School, Family and Community Partnerships. Preparing Educators and Improving School. Boulder: Westwiew Press.

Fernández Enguita, Mariano (2016). La Educación en la encrucijada. Madrid: Fundación Santillana.

Fullan, Michael, Rincón Gallardo, Santiago \& Hargreaves, Andy (2015): "Professional Capital as Accountability". Education Policy Analysis Archives, 23 (15). 
Garreta, Jordi (Coord.) (2017). Familias y escuelas. Discursos y prácticas sobre la participación en la escuela. Madrid: Pirámide.

Garreta, Jordi (Ed.) (2007). La relación familia-escuela. Lleida: Ediciones Universidad de Lleida.

Giró, Joaquín y Andrés, Sergio (2017): "La participación del profesorado en la escuela” en Jordi Garreta (coord.). Familias y escuelas. Discursos y prácticas sobre la participación en la escuela. Madrid: Pirámide.

Giró, Joaquín y Andrés, Sergio (2016): "Instalados en la queja: el profesorado ante la participación de las familias en las escuelas". RASE. Revista de la Asociación de Sociología de la Educación, 9 (3), 334-345. doi: 10.7203/RASE.9.3.8982

Giró, Joaquín y Andrés, Sergio (2014): "Las familias se suben a la Marea Verde. El papel de madres y padres en las movilizaciones educativas". RASE. Revista de la Asociación de Sociología de la Educación, 7 (3), 627-643. doi: 10.7203/RASE.7.3.8746

Giró, Joaquín; Mata, Anna; Vallespir, Jordi y Vigo, Begoña (2014): "Familias y escuelas: los diferentes discursos sobre la participación”. Ehquidad. International Welfare Policies and Social Work Journal, 2, 65-90. doi: http://dx.doi.org/10.15257/ehquidad.2014.0009

Hall, David \& McGinity, Ruth (2015): “Conceptualizing Teacher Professional Identity in Neoliberal times: Resistance, Compliance and Reform". Education policy analysis archives, 23 (88). doi: http://dx.doi. org/10.14507/epaa.v23.2092

Kostogriz, Alex \& Doecke, Brenton (2011): "Standards-based accountability: reification, responsibility and the ethical subject". Teaching Education, 22 (4), 397-412. doi: 10.1080/10476210.2011.587870

Landini, Sonia Regina y Trojan, Rose Meri (2014): "Presupuestos epistemológicos del Informe TALIS: ¿Qué espera la OCDE del profesorado? Profesorado. Revista de curriculum y formación del profesorado, 18 (2), 321-333. http://digibug.ugr.es/handle/10481/33525\#.WLum8G81-M8

Labaree, David F. (2013): "Let's Measure What No One Teaches: PISA, NCLB, and the Shrinking Aims of Education". Teachers College Records, 116. https://web.stanford.edu/ dlabaree/publication2013/ Let's_Measure.pdf

Ley Orgánica 2/2006, de 3 de mayo, de Educación (LOE). Boletín Oficial del Estado, 4 de mayo de 2006, $106,17158-17207$.

Ley Orgánica 8/2013, de 9 de diciembre, para la Mejora de la Calidad Educativa (LOMCE). Boletín Oficial del Estado, 10 de diciembre de 2013, 295, 97858-97921.

Llevot, Nuria y Bernard, Olga (2015): "La participación de las familias en la escuela: factores clave". RASE. Revista de la Asociación de Sociología de la Educación, 8(1), 57-70. doi: 10.7203/RASE.8.1.8761

Maroy, Christian (2013): "Estado Avaliador. Accountability e confiança na Institução Escolar". Revista Educação e Políticas em Debate, 2 (2), 297-318.

Maroy, Christian (2008): “¿Por qué y cómo regular el mercado educativo? Profesorado. Revista de currículum y formación del profesorado, 12 (2). https://www.ugr.es/ recfpro/rev122ART1.pdf

Merchán Iglesias, Francisco (2012): "La introducción en España de la política educativa basada en la gestión empresarial de la escuela: el caso de Andalucía". Archivos Analíticos de Políticas Educativas, 20 (32). http://epaa.asu.edu/ojs/article/view/1059 
Olmedo, Alberto (2008): "De la Participación Democrática a la Elección de Centro: las Bases del Cuasimercado en la Legislación Educativa Española”. Archivos Analíticos de Políticas Educativas, 16 (21). http://epaa.asu.edu/ojs/article/viewFile/46/172

Papadopoulos, Yannis (2010): “Accountability and Multi-level Governance: More Accountability, Less Democracy? West European Politics, 33 (5), 1030-1049. doi: 10.1080/01402382.2010.486126

Parcerisa, Lluís y Verger, Antoni (2016): "Rendición de cuentas y política educativa: Una revisión de la evidencia internacional y futuros retos para la investigación". Profesorado. Revista de currículum y formación del profesorado, 20 (3), 15-51. https://recyt.fecyt.es/index.php/profesorado/article/view/54589

Sarramona i López, Jaume y Rodríguez Neira, Teófilo (2010): "Participación y calidad de la educación", Aula Abierta, 38 (1), 3-14.

Saura, Geo (2015): “Thinks Tanks y educación. Neoliberalismo de FAES en la LOMCE”. Archivos Analiticos de Politicas Educativas, 23 (107). doi: doi.org/10.14507/epaa.v23.2106

Silveira, Héctor (2016): "La participación de las familias en los centros educativos. Un derecho en construcción”. Revista Electrónica Interuniversitaria de Función del Profesorado, 19 (1), 17-29. doi: 10.6018/reifop.19.1.245511

Tiana, Alejandro (2008): "Evaluación y Cambio de los Sistemas Educativos: la Interacción que hace falta". Ensaio: Avaliação e Políticas Públicas em Educação, 16(59), 275-296. http://www.scielo.br/pdf/ ensaio/v16n59/v16n59a07.pdf

Usategui, Elisa y Del Valle, Ana Irene (2009): "Luces y sombras de la función docente desde la mirada del profesorado". Revista Electrónica Interuniversitaria de Función del Profesorado, 12 (2), 19-37. http:/ /www. aufop.com/aufop/uploaded_files/articulos/1248477521.pdf

Viñao, Antonio (2012): "El desmantelamiento del derecho a la educación: discursos y estrategias neoconservadoras". AREAS. Revista Internacional de Ciencias Sociales, 31, 97-107. http:/ / revistas.um.es/areas/ article/view/165031

Whitty, Geoff (2005): “Teachers professionalism in new times”. Journal of In-Service Education, 26 (2), 281-295. doi: 10.1080/13674580000200121

\section{Notas biográficas}

Joaquín Giró Miranda es Profesor Titular Sociología del Departamento de Ciencias Humanas de la Universidad de La Rioja, donde imparte las materias de sociología de la educación en el Máster de Profesorado de Secundaria y en el Grado de Maestro Primaria. Doctor por la Universidad de La Rioja (UR) y licenciado en Ciencias Políticas y Sociología por la Universidad Complutense, ha sido Secretario de la Asociación de Sociología de la Educación (ASE), y del Comité de Investigación 13 (Sociología de la Educación), en la Federación Española de Sociología. Autor de trece libros y coordinador de nueve publicaciones; ha publicado más de veinte artículos en revistas científicas y ha colaborado en treinta y siete obras colectivas. En la actualidad lleva dos líneas de investigación: Sociología de la Educación y Sociología de las Migraciones. Es investigador principal del Grupo de Investigación enclavesocial de la UR. 
Sergio Andrés Cabello es Doctor en Ciencias Políticas y Sociología por la Universidad del País Vasco (UPV). En la actualidad es Profesor Contratado en el Departamento de Ciencias Humanas de la Universidad de La Rioja. Sus áreas de investigación principales se centran en la Sociología de la Educación, el cambio y la estructura social y en el campo de las identidades colectivas. Ha publicado artículos en revistas científicas nacionales e internacionales y ha participado en numerosos congresos y seminarios. Ha sido investigador invitado en la University of Texas at San Antonio (UTSA). Es Vicepresidente de la Asociación Internacional de Ciencias Sociales y Trabajo Social (AICTS) y forma parte del Comité de Investigación de Sociología de la Educación - ASE de la Federación Española de Sociología (FES).

\section{Anexo}

A continuación se recogen los significados de los acrónimos de las citas textuales recogidas en el artículo:

\section{Cuadro 1. Significado acrónimos citas textuales}

\begin{tabular}{|c|c|c|c|}
\hline PARTE DEL ACRÓNIMO & CONCEPTO & CATEGORIAS & \\
\hline \multirow{2}{*}{ Primera } & \multirow{2}{*}{ Tipo de centro } & CEIP & $C$ \\
\hline & & Instituto & 1 \\
\hline \multirow{3}{*}{ Segunda } & \multirow{3}{*}{ Titularidad del centro } & Público & PU \\
\hline & & Privada & $P$ \\
\hline & & Privada Concertado & PC \\
\hline \multirow{15}{*}{ Tercera } & \multirow{15}{*}{ Situación en el centro } & Director & $D$ \\
\hline & & Secretario & $S$ \\
\hline & & Profesor $1^{0}$ y $2^{0}$ Primaria & PP1 \\
\hline & & Profesor $3^{0}$ y $4^{0}$ Primaria & PP2 \\
\hline & & Profesor $5^{\circ}$ y $6^{0}$ Primaria & PP3 \\
\hline & & Profesor $1^{0}$ y $2^{\circ}$ Secundaria & PS1 \\
\hline & & Profesor $1^{0}$ y $2^{\circ}$ Secundaria & PS2 \\
\hline & & AMPA Primaria & Al \\
\hline & & AMPA Secundaria & A2 \\
\hline & & Progenitor $1^{0}$ y $2^{0}$ Primaria & $\mathrm{FPl}$ \\
\hline & & Progenitor $3^{\circ}$ y $4^{0}$ Primaria & FP2 \\
\hline & & Progenitor $5^{\circ}$ y $6^{\circ}$ Primaria & FP3 \\
\hline & & Progenitor $1^{0}$ y $2^{\circ}$ Secundaria & FS1 \\
\hline & & Progenitor $1^{0}$ y $2^{\circ}$ Secundaria & FS2 \\
\hline & & Externo Administración Educativa & TS \\
\hline \multirow{4}{*}{ Cuarta } & \multirow{4}{*}{ Comunidad Autónoma } & Cataluña & CA \\
\hline & & Aragón & AR \\
\hline & & Illes Ballears & IB \\
\hline & & La Rioja & $L R$ \\
\hline \multirow{2}{*}{ Quinta } & \multirow{2}{*}{ Curso escolar } & $2013-14$ & $14 \mathrm{~A}$ \\
\hline & & $2014-15$ & $14 B$ \\
\hline
\end{tabular}

Elaboración propia

Fuente: Proyecto de Investigación "Familias y escuelas. Discursos y prácticas cotidianas sobre la participación en la educación obligatoria" (referencia: EDU2012-32657) 